\title{
Developmental Origins of Biological Explanations: The case of infants' internal property bias
}

\author{
Hernando Taborda-Osorio ${ }^{1} \cdot$ Erik W. Cheries $^{1}$
}

Published online: 18 September 2017

(C) Psychonomic Society, Inc. 2017

\begin{abstract}
People's explanations about the biological world are heavily biased toward internal, non-obvious properties. Adults and children as young as 5 years of age find internal properties more causally central than external features for explaining general biological processes and category membership. In this paper, we describe how this 'internal property bias' may be grounded in two different developmental precursors observed in studies with infants: (1) an early understanding of biological agency that is apparent in infants' reasoning about animals, and (2) the acquisition of kind-based representations that distinguish between essential and accidental properties, spanning from animals to artifacts. We argue that these precursors may support the progressive construction of the notion of biological kinds and explanations during childhood. Shortly after their first year of life, infants seem to represent the internal properties of animates as more central and identity-determining that external properties. Over time, this skeletal notion of biological kinds is integrated into diverse explanations about kind membership and biological processes, with an increasingly better understanding of the causal role of internal properties.
\end{abstract}

Keywords Internal properties $\cdot$ Infancy $\cdot$ Explanation · Biology

Hernando Taborda-Osorio

htaborda@psych.umass.edu

Erik W. Cheries

echeries@psych.umass.edu

1 Department of Psychological and Brain Sciences, University of Massachusetts, Amherst, Tobin Hall, 135 Hicks Way, Amherst, MA 01003, USA

\section{Introduction}

Generating explanations of biological phenomena is a widespread activity across all ages and cultural groups (Medin \& Atran, 1999). The entities that populate the natural world exhibit a variety of shapes, colors, functions, and characteristic behaviors that both children and adults automatically attempt to understand in terms of their root causes. One of the overarching goals of research in this area has been to identify the underlying structure of the causal-explanatory framework that supports these intuitions.

Many of the explanations we construct refer to causal mechanisms that are heavily biased towards particular kinds of features. For example, causal explanations in the biological domain appear to be powerfully biased towards 'deeper,' unseen features as opposed to those that exist on the surface (Ahn, 1998). Such features are commonly described as 'nonobvious' or 'internal' properties, since they evade immediate perception by existing either beneath the surface (e.g., physical insides) or by being caused by unobservable internal mechanisms (e.g., sound properties). This distinctionweighting 'internal' over 'external' features - is apparent in at least two types of biological explanations: explaining general biological functions and explaining species-typical properties. Examples of the former type involve the explanation of growth, movement in animals, and the recovery of health, among other functions (Inagaki \& Hatano, 2004). Adults have an overall understanding of how physical internal properties such as muscles and bones are causally relevant in the generation of motion, or that internal fluids are necessary to keep animals and plants alive. Adults also understand that the brain is like a container holding thoughts, memories, and ideas that are also somehow related to different personality types (Gottfried, Gelman, \& Schultz, 1999). Additionally, explaining species-typical properties such as differences in 
body shape, color, and behavior also requires an understanding of how physical internal features are causally relevant in the generation of those properties (Rehder, 2003). For example, many individuals in Western cultures attribute to DNA a preeminent causal role in the generation of species-typical properties (Dar-Nimrod \& Heine, 2011). Furthermore, some physiochemical processes may be invoked in order to explain why animals and plants display particular properties rather than others. In both types of explanations - general biological functions and species-typical properties-adults are biased toward representing biological entities as possessing an underlying structure with causal potency.

The main goal of the current paper is to outline one proposal about the developmental origins of this internal property bias and its role in the elaboration of biological explanations. Of course, children eventually learn a great deal about the internal physical properties of animals and plants and miscellaneous biological processes through schooling. For example, they are explicitly taught about the internal causes of important bodily functions. However, an increasing number of studies have also documented a bias toward internal properties in young children who lack exposure to these ideas through formal education (Simon \& Keil, 1995; Diesendruck, 2001; Diesendruck \& Peretz, 2013). For example, 5-year-old children that weigh causal features (e.g., blood type) over effect features (e.g., skin color) in categorization judgments, in general, do so only when those causal properties are presented inside the organism (Meunier \& Cordier, 2009). Children this age appear biased to represent an animal's internal physical properties as more causally responsible and diagnostic of category membership than any of its external properties. Such internal property biases are symptomatic of 'biological essentialism,' an assumption that living things possess hidden causal forces that maintain an individual's identity over time and change, which is widespread across children's biological explanations in a variety of cultures (Waxman, Medin, \& Ross, 2007).

Where does this internal property bias in children come from? Our proposal is that two different developmental precursors presenting during infancy conspire to develop this bias: first, an ontological distinction between kinds and classes that supports the idea of hidden causal factors when thinking of non-accidental categories in the world (Cimpian \& Erickson 2012; Gelman \& Hirschfeld, 1999), and second, the ascription of biological properties to self-propelled and agentive entities (Gelman, 1990). In the first two sections of this paper, we describe the ways in which each of these precursors draws infants' attention toward non-obvious properties from very early on. In the third section, we explain how these precursors jointly contribute to the emergence of an internal property bias that causes infants to weigh the internal properties of animate objects more heavily than external ones, laying the foundation for the concept 'biological kind.' In the fourth section, we argue how this skeletal representation may give rise to the more elaborated intuitive theory of biological essentialism. In the final section, we describe how this early internal property bias becomes elaborated through a protracted developmental process into a fully mature understanding of the biological role that physical inside properties play (e.g., Rhodes \& Gelman, 2009).

\section{Kind Representations in Infancy}

The human conceptual system makes an important distinction between two types of categories: kind categories and class categories (Prasada, Hennefield, \& Otap, 2012). Kinds and classes differ from each other based on both the category structure (Estes, 2003) and the type of inferences they support (Rips, 2011). At the level of category structure, kind concepts are represented as non-accidental categories supported by rich causal-explanatory networks (Keil, 1995). The set of correlated properties that particular instances of a kind display are explained by positing causally potent properties that lie beneath the surface (Ahn, Taylor, Kato, Marsh, \& Bloom, 2013; Kripke, 1980; Medin \& Ortony 1989; Putnam, 1975; Quine, 1969). Such non-observable, or so-called 'non-obvious,' properties are referred to as 'defining' or 'essential' features. By contrast, class concepts are represented as accidental categories, picking out objects from different kinds, and with a relatively shallow structure (Shipley, 1989). These differences in category structure have consequences in the type of inferences that people can carry out. For example, it has been shown that adults and older children categorize biological kinds based on underlying and unobservable properties rather than visible features (Ahn, Gelman, Amsterlaw, Hohenstein, \& Kalish, 2000; Rehder \& Kim, 2006). Adults may sometimes rely on external properties to categorize biological kinds but only when they are causally connected to non-visible properties (Rehder \& Kim, 2009). Adults also find kind membership to be more central to the representation of individual identity than superficial features (Hirsch, 1982).

The way children and adults represent kind concepts across several domains (e.g., artifacts, living entities, and human kinds) exhibits a high degree of developmental continuity (e.g., Gergely \& Jacob, 2012). This continuity is manifest within the first two years of life in at least two types of inferential processes: categorization and individuation.

\section{Kind-based Categorization}

Some developmental studies indicate that infants use kind information in categorization tasks. For example, 11-monthold infants are able to categorize different animal figures (e.g., a giraffe and a crocodile) and different furniture figures (e.g., a 
chair and a bed) as two contrasting and global kinds (Pauen, 2002), even though the degree of similarity between kinds was lower than the degree of similarity within kinds (e.g., Mandler \& McDonough 1993; 1998). Infants this same age have also been shown to categorize artifacts based on relevant functional information rather than their overall perceptual similarity (Trauble \& Pauen, 2007), but only when object and function are causally connected (Booth, 2008; Booth, Schuler, \& Zajicek, 2010; Gopnik \& Sobel, 2000). In this way, infants, like adults, seem to represent function as a critical property of artifact kinds (Hernik \& Csibra, 2009), and use kind membership as a more reliable source of information to categorize animals than perceptual similarity.

In kind-based categorization, representing surface features is important to the extent that it diagnoses the presence of 'deeper' non-observable properties (Rehder, 2007). Evidence of this type of 'diagnostic reasoning' in infancy has been observed using artifacts. For example, studies using a longitudinal methodology have shown that 17-month-old infants exhibit a 'shape bias' when categorizing artifacts, but only when they perceive shape as causally important for performing a particular function (Ware \& Booth, 2010). Infants learn that the shape of a spoon is a relevant feature if the spoon is used to scoop sand from a bucket but not when it is used to cut something. Crucially, after 6 weeks of training, these children also transferred this 'shape bias' to new artifacts. Other studies with younger infants have demonstrated that 9-month-olds are biased to use an artifact's shape rather than its color to distinguish the referent of new nouns (Dewar \& Xu, 2007). However, when shape and the display of a non-obvious property (e.g., sound) are pitted against each other, infants believe that different words refer to objects with different non-obvious properties (Dewar \& Xu, 2009). These results suggest that shape is a salient property early on because it is a reliable cue of more central nonobservable properties. Thus, infants seem to have the intuition that some visible properties are particularly relevant for categorizing objects in virtue of being more diagnostic of kind membership (Graham \& Diesendruck, 2010).

\section{Kind-based Object Individuation}

In addition to specifying category membership, kind representations can also be used for identifying particular instances of objects in those categories and tracing their individual identity over time (Xu, 2005). Particularly when an object's spatiotemporal history is ambiguous, adults use kind-based information in order to judge whether or not it is the same individual seen previously. For example, adults perceive a magician turning a white dove into a white rabbit as an impossible transformation in large part because they are different kinds of things. The difficulty in identifying the two animals as belonging to the same category leads people to represent two different and persistent individuals in the display. This process is called kind-based individuation (see Carey \& Xu, 2001 for a review).

Developmental studies have revealed that, by 12 months of age, infants seem capable of individuating objects based on kind information. When infants this age witness two different kinds of objects (e.g., a duck and a ball) appearing sequentially from behind a screen in a spatiotemporally ambiguous situation, they seem to represent two individual entities rather than just one changing its appearance (Xu \& Carey, 1996). This inference does not depend merely on the surface property differences (e.g., shape, color) across stimuli in these tasks, since infants appear relatively insensitive to the salient property differences between a red cup and a blue cup, which are both exemplars of the same kind (Kingo \& Krojgaard, 2011; $\mathrm{Xu}$, Carey, \& Quint, 2004). Indeed, an important characteristic of kind-based theories of individuation is that non-obvious properties should be privileged over external properties, while representing an object's identity over time. Evidence for this bias has been observed in different domains. For example, when 10-month-olds witness a person demonstrating two different functions on the same artifact at different times in a typical individuation task, they seem to represent two objects involved in the event, demonstrating an early one-to-one mapping between artifact and function (Futo, Teglas, Csibra, \& Gergely, 2010). Similarly, when 11-month-olds witness an agent perform two different socio-moral actions (e.g., helping and hindering) in an individuation task, they tend to represent two identical-looking agents behind the screen rather than one agent who changes dispositions (Taborda-Osorio \& Cheries, 2016). In this way, infants seem to be biased away from surface-level features and towards deeper non-obvious properties, such as an artifact's function or an agent's social disposition, when tracing the individual identity of objects over time.

Overall, studies in categorization and individuation suggest that object kinds are represented as possessing an underlying structure that is more identity-determining than obvious, surface-level features like shape and color. There are two points worth underscoring in the current discussion. First, this is a bias that emerges early on in life and may help facilitate the mapping between words and referents (Csibra \& Shamsudheen, 2015). Second, it seems to be a relatively general bias, insofar as a similar pattern of results emerges when looking at how infants represent the identity of artifacts, living entities, and some social categories. The global nature of this bias indicates that ascribing non-obvious properties may be a basic human mode of understanding the world, affording infants the flexibility to determine the type of properties and causal mechanisms that may be relevant in each domain. 


\section{Representations of Animate Agents in Infancy}

The concept 'living thing' undergoes a substantial conceptual change over the course of childhood (Carey, 1985). A mature understanding of living things, including both animals and plants (with humans as a subset of animals), is not achieved until around 7-8 years of age (Johnson \& Carey, 1998). However, younger children seem to possess a clear and relatively rich conceptual distinction between animate and inanimate objects (Hatano \& Inagaki, 1999). For example, children as early as 5 years of age know that animate and inanimate objects belong to different ontological categories (Keil, 1989). When children are questioned about an object transforming its identity to another, they are more likely to deny the possibility if the change is across ontological categories (e.g., a toy bird to a real bird), than within an ontological category (e.g., a horse to a zebra). Additionally, 5-years-olds also know that only animate entities display goal-oriented autonomous movements (Opfer, 2002). When these children are shown videos of a blob displaying either an aimless self-propelled movement or a movement that is both self-propelled and goal-oriented, they claim that only the latter possesses biological properties (e.g., needing to eat). This result indicates that selfpropelled movement alone is not a sufficient cue for inferring animacy. Instead, goal-oriented movement seems to be a central component of children's concept of living things, which is initially applied only to animals and then extended to both animals and plants (Opfer \& Siegler, 2004).

The distinction that children draw between animate and inanimate entities also supports differences in internal structure (Simons \& Keil, 1995). In particular, children expect the insides of animate and inanimate objects to differ (Gottfried \& Gelman, 2005). For example, preschool-aged children tend to choose the appropriate type of insides for animals, plants, and machines (e.g., a heart inside animals and wires inside machines). Children also expect a higher degree of similarity in the type of internal properties that animate objects possess compared to the internal properties of inanimate objects (Gelman, 1990). These results indicate that, for children, animals differ from inanimate objects in deep, fundamental ways.

Studies with infants suggest a large amount of developmental continuity in terms of the skeletal representations that support the animate-inanimate distinction (Gelman, 2009). For example, infants as young as 9 months of age are able to generalize previously observed actions to other objects in the appropriate ontological category (Mandler \& McDonough, 1996; 1998). When infants observe the experimenter using a key to start a car, they reproduce the action with an airplane but not with a bird, even though they look very similar (Mandler, 2004). Convergent evidence of this ontological distinction comes from work using an object individuation paradigm. In one such study (Surian \& Caldi, 2010), 10-month-old infants observed two contrasting objects emerging from behind a screen at different times. One object displayed typical featural and dynamic animate information (e.g., a bee flying), and the other typical inanimate information (e.g., a cup being moved by a hand). Looking time measures indicated that infants succeeded in individuating two objects behind the screen. Crucially, infants failed to individuate two objects from one another that were sampled from within each ontological category (e.g., a bee and a caterpillar), suggesting that infants this age lack more nuanced taxonomical categories.

In addition to making a general ontological distinction, do infants make biological attributions about animate objects? As we have seen, the perception of goal-directed movement is a central component of older children's concept 'animate agent' (Opfer, 2002), and this input leads children to attribute the presence of physical internal properties (Gelman, 1990). If this reasoning is grounded by initial skeletal principles, infants may demonstrate similar inferences, although less rich in details. In a recent study (Setoh, Wu, Baillargeon, \& Gelman, 2013), 8-month-old infants were shown a box on a stage in three different conditions: The box displayed a self-propelled movement only, agentive cues only (e.g., engaged in a "conversation" with the experimenter), or both self-propelled movement and agentive cues. After familiarizing infants with one of these events, the experimenter lifted the can revealing it to be closed at the bottom or empty. The results of this experiment showed that infants expected the can to have filled insides only in the self-propelled and agentive conditions. As with older children, the perception of self-propelled movement itself does not trigger the attribution of physical internal properties. Although infants this age conceptually distinguish agents from recipients of movement (Leslie, 1984),based on the notion of transmission of force (Leslie, 1994), it seems not to imply a distinction between animate and inanimate objects. Additionally, the absence of biological expectations in the agentive cues-only condition suggests that the attribution of internal psychological properties does not necessarily entail the attribution of internal physical properties. Thus, the animate-inanimate distinction seems to be conceptually different from the infants' system of psychological reasoning, and infants require evidence of both kinds of properties to infer that the entity should possess physical insides.

The Setoh et al. (2013) findings may reveal the operation of an 'innards principle' in infancy (Gelman, 1990), in which physical internal properties are attributed to an animal in order to explain both its self-propelled movement and agency. Additionally, some authors have speculated that the detection of animals with physical internal properties may be the result of an evolutionary adaptation in a prey-predator context and the detection of possible sources of valuable nutrients (Barrett, 2005; Opfer, 2002; Setoh et al., 2013). Indeed, developmental studies show that fitness-relevant mode of interactions, such as "chasing" and "helping", are perceptually salient for infants (Frankenhuis \& Barrett, 2013). 
Both aforementioned precursors-kind-based representations and the animate-inanimate distinction - have the power to draw infants' attention away from surface-level features and towards the non-obvious properties possessed by an entity. However, unlike the very general biases that underlie infants' kind-based representations, the animate-inanimate distinction seems to focus infants' attention towards a particular type of non-obvious property: physical insides. In the biological domain, causal explanations are strongly tied to physical insides over any other, even those that are also represented as nonobvious but internal properties in other domains, such as goals or intentions in the psychological domain and an internal source of energy in the physical domain. Thus, the main question of the current paper addressed in the next section is how infants come to represent the physical internal properties rather than other properties as a central component in diverse biological explanations, and the conceptualization of animate entities overall.

\section{The Internal Property Bias in Infancy}

The evidence presented so far shows that infants in the first year of life possess (1) a domain-general bias to represent kind membership as being caused by non-obvious properties, and (2) a domain-specific process that attributes physical insides to animate entities. Our proposal is that together these two cognitive processes provide the foundations for the concept biological kind and the progressive construction of a biological causal-explanatory theory (cf. Inagaki \& Hatano, 2002). Namely, children eventually represent an animal's internal features as a biological property that is causally related to determining its kind membership. The critical point, however, is that neither of these two processes necessarily licenses this belief in isolation from one another. On the one hand, young infants might attribute physical insides to an animate agent without drawing any connection between those properties and the agent's kind identity. On the other hand, infants' bias towards non-obvious properties may highlight many different types of features that could be used to determine an agent's kind identity (e.g., sound, movement, an intrinsic source of energy, intentions, physical insides, etc.). In this way, a mature understanding of the importance of physical insides in biology requires both of these processes in combination. For instance, the array of non-obvious properties that may determine an object's kind is significantly constrained by the domainspecific expectations infants possess at a young age. Since infants expect that only self-propelled and agentive entities (i.e. animals) possess physical internal properties, they may infer that physical insides, per se, rather than other unobservable properties are a particularly relevant non-obvious property for biological kinds. This inference would not be possible if infants lacked a domain-specific expectation of animals having physical internal properties, as they have little or no information about the insides of living kinds. Thus, by highlighting the importance of non-obvious properties, kind representations may lead children to represent physical internal properties as a central feature in the conceptualization of animate entities. How early do these two cognitive processes coalesce in development?

One way of diagnosing whether an agent's 'insides' are represented as a biological kind property, and not just as a characteristic feature of animate objects, is to test whether an agent's physical insides are viewed as more identitydetermining than other properties in individuation and categorization tasks. Some recent experiments have examined how infants in the second year of life represent the role of visible physical internal properties when categorizing and keeping track of the identity of animate and inanimate objects. For example, in an object-examining task, 14-month-olds categorize inanimate objects based on their most salient external features, but, when those same objects are presented with animacy cues (e.g., with eyes on the top), infants were more willing to categorize them based upon less obvious physical internal properties (Welder \& Graham, 2006). Other studies have shown an early sensitivity to physical internal properties in representing an object's identity. For instance, 14-montholds were familiarized with a self-propelled transparent toy with contrasting colors on the inside and the outside, and eyes on the top (Newman, Herrmann, Wynn, \& Keil, 2008). Afterwards, two different objects, one with a similar physical inside and one with a similar outside, were displayed in front of the child. The results revealed that the majority of infants picked out the object with the same-color physical insides. Crucially, when motion was externally generated (as opposed to self-propelled), infants did not show a larger preference for one or the other toy. It seems that perceiving animate motion prompts infants to weigh an object's physical internal properties more heavily than its external properties.

Infants display a perceptual bias toward the physical internal features of animate agents. One interpretation of this phenomenon is that infants represent these agents as biological kinds such that their internal properties are treated as more causally related to its category membership. If this interpretation is correct, then infants should use perceived differences in internal properties to establish representations of new individuals. This prediction was tested using an object individuation paradigm with 13-month-olds who were shown animate objects containing visible internal and external features that possessed different combinations of contrasting colors (TabordaOsorio \& Cheries, 2015). Like other individuation experiments of this type (e.g., Van de Walle, Carey, \& Prevor, 2000; Feigenson \& Carey, 2003), infants observed two of these objects appear and disappear from a box at different times with no clue to their numerical identity besides the featural information they displayed. The results showed that, 
when infants observed two self-propelled objects with the same-color insides but different-color outsides, they seemed to represent only one enduring entity involved in the event. By contrast, when infants witnessed two objects with differentcolor insides but the same-color outsides, they represented two individuals. Importantly, infants did not make use of the physical internal property differences they observed when the two objects lacked animacy cues (e.g., eyes and self-propelled motion). This pattern of results suggests that infants treat biologically-based cues, such as an agent's physical insides, as a powerful criteria for representing an agent's unique identity (Taborda-Osorio \& Cheries, 2015).

In addition to determining kind-identity, other evidence suggests that infants may interpret an agent's physical internal properties as being causally related to the characteristic behavior it displays. When 14-month-olds observe an animate transparent object displaying an idiosyncratic motion (e.g., moving up and down or moving side to side), they expect objects with the same color inside but not those with the same color outside to exhibit the same type of behavior (Newman, et, al., 2008). Even more abstract behaviors, such as an agent's sociomoral behavior toward another, seems to be more readily associated with internal than external properties by infants. For example, research has determined that infants correctly interpret an agent's social disposition towards another as 'helpful' or 'unhelpful' depending on its pattern of interaction, such as helping or hindering an agent's goal of successfully climbing a hill (Hamlin, Wynn, \& Bloom, 2007; Kuhlmeier, Wynn, \& Bloom, 2003). Studies using an identical social scenario have shown that infants more readily associate such social behaviors with an agent's inside properties rather than their outside ones. First, infants ignore superficial changes to a character's surface appearance and expect a 'helpful' character which is painted like an 'unhelpful' character to continue being helpful and vice versa (Cheries, Newman, \& Wynn, 2015). Second, infants who observe transparent objects displaying either prosocial or an antisocial behaviors show a preference to reach for the object that matches the physical internal property of the prosocial agent (Taborda-Osorio, Lyons, \& Cheries, 2016). These results indicate that infants might use biological criteria, like an agent's physical internal property, to distinguish different social kinds. Physical internal properties may be intuited by infants to be more causally related to a character's social behavior.

Overall, the previous studies show that there is an early appreciation of the relationship among physical internal properties, identity, and category membership in agentive entities. This type of complex mental representation cannot be the result of either a domain-specific mechanism for biological entities or a domain-general mechanism of kind-based representations alone. On the one hand, a domain-specific account in terms of an "innards principle" specifies the existence of physical internal properties in self-propelled and agentive entities, but it cannot fully explain why from very early on physical internal features are treated as kind-relevant properties that carry causal power. On the other hand, a domaingeneral account of kind-based representations allows infants to represent some categories as endowed with a hidden causal power, but it cannot by itself center infants' attention on a specific non-obvious property as this may vary across different categories. However, a plausible developmental explanation is that these two processes eventually combine. What starts as an early bias towards an animal's physical internal properties is gradually elaborated until it becomes a kindrelevant property and the foundation of categorical identity when reasoning about animals. The evidence presented above suggests that this elaboration may start as early as the second year of life, as soon as the two constituent biases emerge or soon thereafter. For instance, this developmental model does not imply a strictly sequential emergence where one assumption, the internal property bias, emerges from two precursors only after a developmental delay. Instead, the representation of animals as biological kinds may be constructed out of both precursors as a type of emergent property as soon as infants are exposed to them. Thus, the representation of physical internal properties in animals as kind-relevant may turn out to be a useful assumption to explain the diversity of shapes and behaviors that infants encode.

\section{The Internal Property Bias and the Development of Biological Essentialism}

The internal property bias in infancy described above may have lasting consequences for how children and adults conceptualize biological kinds and explain biological phenomena. Indeed, unlike artifacts, animals are construed as natural kinds, defined in terms of an essential, intrinsic and objective property, meaning that whatever determines their kind membership is a more permanent property and more resistant to change over time (Kelemen \& Carey, 2007; Rips, 2011). For instance, adults believe that external transformations may lead to changes in category membership for artifacts but not necessarily for animals (Braisby, Franks, \& Hampton, 1996; Keil, 1991).

If children are biased toward representing animals' physical internal structure as a biological kind property, then the identity of animate objects should be viewed as more intrinsic, since internal properties are harder to change than external properties. Studies observing this pattern reflect how children seem to construe biological kinds, but not artifacts, as natural kinds. For instance, 4-year-olds believe animals that belong to the same category share internal, non-observable properties to a greater extent than physical or perceptual properties (Gelman \& Markman, 1986). Relatedly, 3- to 5-year-old children believe animals that share the same internal properties 
share the same category membership, even to some extent overriding perceptual dissimilarities (Diesendruck, 2001; Diesendruck, Gelman, \& Lebowitz, 1998; Diesendruck \& Peretz, 2013). In both inductive inference and categorization tasks, children display a greater reliance on physical internal properties for animals than for artifacts. However, in tasks where the causal properties of complex artifacts are demonstrated in front of the child and the internal properties are visible, 4- and 5-year-old children use internal but not external properties as the basis for categorization (Sobel, Yoachim, Gopnik, Meltzoff, \& Blumenthal, 2007; Sobel \& Buchanan, 2009). This demonstrates how the internal properties of some non-living entities may be privileged over external features to the extent that they reflect some sort of underlying causal power (Gopnik \& Nazzi, 2003). Nevertheless, the early animate-inanimate distinction, and in particular the representation of animals as entities with internal structure, seem to bias infants' and older children's attention toward the internal properties of biological agents to a greater extent.

According to some authors, biological categories that are represented as possessing intrinsic and objective properties reflect a kind of psychological essentialism (Carey, 2009; Gelman, 2003). Under this framework, biological categories have a rich inductive potential whose members possess nonvisible, internal essences that are represented as being responsible for its surface features. Some researchers have proposed that children represent biological and some social categories in these terms (Cimpian \& Solomon, 2014, Gelman \& Hirschfeld, 1999; Keil, 1989). For instance, an unspecified internal essence in the category lion would be responsible for a wide variety of morphological and behavioral regularities, such as having manes and hunting gazelles. This type of complex reasoning seems to be grounded in some explanatory biases. Namely, children as young as 4 years of age explain regularities in their environment as a function of inherent features, rather than extrinsic properties or historical facts (Cimpian \& Solomon, 2014). For young children those inherent features can have an open-ended output because they lack relevant semantic knowledge, and thus the inherent features that explain a regularity can be undetermined. Eventually, this open-ended output is elaborated or filled with the assumption of an essence to explain regularities in biological entities. This explanation is supplemented by the consideration of early developmental biases. As was shown before, infants have the notion that animate objects possess internal properties and an internal source of energy that later on in development may serve as a placeholder to attribute real internal essences in animals (Cimpian \& Solomon, 2014).

The roots of psychological essentialism may be found early in development and may be richer than the attribution of physical internal properties to biological kinds. As we have argued, in the first years of life children may represent animals' physical internal properties as both a causal and an identity- determining feature through the joint operation of domaingeneral and domain-specific mechanisms. Infants' bias to weigh the physical internal properties of self-propelled and animate objects more heavily than external features constitutes the basic foundation for biological essentialism. However, this initial notion about the relationship between physical internal properties, causality, and identity is far from being a full biological essentialist theory. For this to be achieved, children have to enrich and differentiate their basic-level concepts, which is a longer process for biological than for artifact concepts (Mandler, 2004). Furthermore, children must develop the ability to view physical internal properties as causally connected to both the behavioral and morphological features of living things. In other words, development must move from the broad notion that internal properties are more diagnostic of identity than external properties in animate objects to the notion of an internal essence that gives rise to observable similarities in members of a particular kind. This final product is a theory of biological essentialism.

\section{Developmental Changes in Children's Biological Explanations}

Although the early internal property bias presented above is a central component in the children's intuitive biological knowledge, it undergoes important elaboration along development toward the construction of a biological causal-explanatory theory. There are at least two notions that need to be developed along childhood in order to reach a mature understanding about the role of physical internal properties in a biological framework. First, children should appeal to the causal role of physical internal properties when explaining both the general biological functions of animate objects and their speciestypical properties. For example, adults know that different internal mechanisms are causally related to different vital functions, and that different types of insides are related to different species. Second, the causal network that connects internal and external properties should be understood in terms of a mechanistic explanation.

Several studies have noticed the relatively poor understanding of both notions in preschoolers, and a conceptual change taking place between around 4 and 8 years of age. Thus, young children do not seem to understand the causal role that internal properties have in the generation of external morphological features of both animals and plants (Meunier \& Cordier, 2009). For instance, 5-year-old children, but not 4-years-olds, classify objects as belonging to the same category if they share internal properties (e.g., a substance inside) and they are described as causally responsible for external features (e.g., red petals). Therefore, 5-years-olds expect external morphological features to be causally connected 
with internal properties. This contrasts with younger children who believe that internal properties are more identity-determining but seem to have poorer knowledge about their causal relevance in the expression of biological traits (Meunier \& Cordier, 2009).

The lack of knowledge about the causal role of internal properties in preschoolers has also been observed in the pattern of explanations of general biological processes, such as movement and growth (Gelman \& Gottfried, 1996; Gottfried \& Gelman, 2005). When 5-year-olds are asked to choose if animals use their insides, their own energy, or a particular part inside of them to move, they typically choose "their own energy" as the best answer. Additionally, when 4- and 5year-old children are presented with a scenario where a brain or other insides part is transplanted across animals of different species, they do not believe the individual and typical-species properties can change as a result of this operation (Gottfried, Gelman, \& Schultz, 1999). However, children understand that internal properties are necessary for animals' correct functioning. For instance, children deny that a dog can still be a dog and bark if all the insides are removed (Gelman \& Wellman, 1991). Similarly, although children do not believe that something inside is the cause of movement, they expect more internal parts for moving than for nonmoving objects (Gelman, 2003). Therefore, young children represent physical internal properties as a necessary component of typical biological processes rather than a sufficient cause (Gelman, 2003; Gottfried \& Gelman, 2005).

A mechanistic explanation about bodily functioning seems also to emerge relatively late in development, as the role of autonomous operation of internal properties becomes increasingly central through formal education. For example, 8-yearold children, but not 4-year-olds, believe that bodily functions (such as growth and movement) are directly caused by insides or internal parts (Gottfried \& Gelman, 2005), and endorse mechanistic explanations when confronted with general biological functions (e.g., breathing to take in oxygen and changing it into waste carbon dioxide; Inagaki \& Hatano, 2004). Furthermore, 9- to 10-year-old children who learn about DNA may appeal to genes when justifying their intuitions about the origins of physical and behavioral properties (Heyman \& Gelman, 2000), and eventually endorse a form of genetic essentialism to explain the transmission of various traits (see Dar-Nimrod \& Heine, 2011 for a review).

How do older children come to understand the role of internal physical properties in animate objects in this causal and mechanistic way? Although more research is necessary to understand this developmental process, and formal education is certainly key, the early representation of animate entities as biological kinds may be instrumental in this achievement. In particular, the notion of internal physical properties as a kind property may serve as a causal placeholder. Young children may believe that something intrinsic to the animal's body is, in an unspecified manner, necessary to explain the animal's characteristic features and behaviors, and is responsible for kind membership. The way this causal notion is instantiated when children are asked to explain different biological phenomena takes varied forms, and in some cases seems to resemble prescientific theories. For example, when 4-year-old children are asked to explain the transmission of properties in plants, they choose internal rather than external mechanisms as the best option (Springer \& Keil, 1991). Apparently, children endorse a kind of "Gemmulic" theory of inheritance, appealing to tiny pieces inside the parent transmitted to offspring with a copy of a target property (Newman \& Keil, 2008).

The skeletal causal notion of internal properties is also elaborated by children when explaining general biological functions. Some studies show that 5- and 6-year-old children in diverse cultures understand that internal parts are necessary to sustain an exchange of vital force or energy with the external environment (Inagaki \& Hatano, 1993). For example, children find it more reasonable to explain the intake of food as a process where an internal organ takes in energy from the food than as a process where the internal organ transforms the food for the body (Inagaki \& Hatano, 2004). The intuitive use of vitalistic explanations seems to have two complementary components: the reliance on an exchange of vital force, and the ascription of "agency" to internal organs (Morris, Taplin, \& Gelman, 2000). Thus, children endorse almost as often explanations referring to both an intake of energy and an organ wanting to fulfill a function (e.g., we eat because our stomach wants to take in food). However, children do not attribute other mental processes, such as thinking, to internal organs or overall finding bodily functions less compelling, than intentional explanations (e.g., we eat because we like tasty food). Therefore, the reliance on an organ's intentions in vitalistic explanations may signal some children's understanding of the autonomous functioning of internal organs, which is a hallmark of more mature physiological explanations.

\section{Conclusions}

Psychological research has demonstrated that the way people provide explanations is constrained by domain-specific theory-like structures of knowledge (Gelman, 2009; Tenenbaum, Griffiths \& Kemp, 2006). For example, people's intuitive knowledge about physical events is constrained by the laws of mechanics (Leslie, 1984), and intuitive psychological knowledge follows the principle of rationality (Gergely \& Csibra, 2003). Similarly, biological knowledge is seemingly constrained by the relative weight people attribute to an organism's internal and external properties, with its 'insides' being much more central in the elaboration of biological explanations (Ahn, 1998). In this paper, we have argued that a skeletal form of this internal property bias exists in infancy 
and is derived through two distinct sources: (1) the use of hidden and non-obvious properties for determining kind membership, and (2) through the attribution of internal structure to agentive and self-propelled entities.

The early internal property bias may constrain the elaboration of a biological causal-explanatory theory in at least two different manners. First, the early representation of internal physical properties as kind-relevant may lead young children to construe an intuitive theory of biological essentialism, and, thus, an agent's 'insides' may be conceptualized as a physical instantiation of biological essences (Newman \& Keil, 2008), or at least something conceptually proximate (Gelman, 2003). Accordingly, young children believe that internal physical properties are more privileged than external properties when explaining species-typical features in animals. Second, the representation of internal physical properties as kindrelevant may also serve as a causal placeholder for developing an initial understanding of how biological phenomena work. Thus, young children are biased to represent animals' physical internal properties as a necessary, although not sufficient, component when explaining general biological processes. For instance, children believe that the working of internal organs sustains the vital force involved in growth and movement. In both cases, the animals' internal structure plays a central causal role in the children's mind that may give rise to a more elaborated and scientifically correct biological explanations.

\section{References}

Ahn, W. (1998). Why are different features central for natural kinds and artifacts?: The role of causal status in determining feature centrality. Cognition, 69, 135-178.

Ahn, W., Gelman, S. A., Amsterlaw, J. A., Hohenstein, J., \& Kalish, C. W. (2000). Causal status effect in children's categorization. Cognition, 76, B35-B43.

Ahn, W.-K., Taylor, E. G., Kato, D., Marsh, J. K., \& Bloom, P. (2013). Causal essentialism in kinds. The Quarterly Journal of Experimental Psychology, 66(6), 1113-1130.

Barrett, H. C. (2005). Adaptations to predators and prey. In D. M. Buss (Ed.), The Handbook of Evolutionary Psychology (pp. 200-223). Hoboken: John Wiley.

Booth, A. E. (2008). The cause of infant categorization? Cognition, 106, 984-993.

Booth, A. E., Schuler, K., \& Zajicek, R. (2010). Specifying the role of function in infant categorization. Infant Behavior and Development, 33(4), 672-684.

Braisby, N., Franks, B., \& Hampton, J. (1996). Essentialism, word use, and concepts. Cognition, 59, 247-274.

Carey, S. (1985). Conceptual change in childhood. Cambridge: MIT Press.

Carey, S. (2009). The Origin of Concepts. USA: Oxford University Press.

Carey, S., \& Xu, F. (2001). Infants' knowledge of objects: Beyond object files and object tracking. Cognition, 80, 179-213.
Cheries, E. W., Newman, G. E., \& Wynn, K. (2015). A wolf in sheep's clothing? Infants ignore changes in external appearance when tracking social others. Manuscript submitted for publication.

Cimpian, A., \& Erickson, L. (2012). Remembering kinds: New evidence that categories are privileged in children's thinking. Cognitive Psychology, 64, 161-185.

Cimpian, A., \& Salomon, E. (2014). The inherence heuristic: An intuitive means of making sense of the world, and a potential precursor to psychological essentialism. Behavioral \& Brain Sciences, 37, 461527.

Csibra, G., \& Shamsudheen, R. (2015). Nonverbal generics: Human infants interpret objects as symbols of object kinds. Annual Review Psychology, 66, 689-710.

Dar-Nimrod, I., \& Heine, S. J. (2011). Genetic essentialism: On the deceptive determinism of DNA. Psychological Bulletin, 137, 800818.

Dewar, K., \& Xu, F. (2007). Do 9-month-old infants expect distinct words to refer to kinds? Developmental Psychology, 43, 1227-1238.

Dewar, K., \& Xu, F. (2009). Do early nouns refer to kinds or distinct shapes? Evidence from 10-month-old infants. Psychological Science, 20(2), 252-257.

Diesendruck, G. (2001). Essentialism in Brazilian children's extensions of animal names. Developmental Psychology, 37(1), 49-60.

Diesendruck, G., Gelman, S. A., \& Lebowitz, K. (1998). Conceptual and linguistic biases in children's word learning. Developmental Psychology, 34, 823-839.

Diesendruck, G., \& Peretz, S. (2013). Domain differences in the weights of perceptual and conceptual information in children's categorization. Developmental Psychology, 49, 2383-2395.

Estes, Z. (2003). Domain differences in the structure of artifactual and natural categories. Memory and Cognition, 31(2), 199-214.

Feigenson, L., \& Carey, S. (2003). Tracking individuals via object-files: Evidence from infants' manual search. Developmental Science, 6, $568-584$.

Frankenhuis, W. E., \& Barrett, H. C. (2013). Design for learning: The case of chasing. In M. D. Rutherford \& V. A. Kuhlmeier (Eds.), Social perception (pp. 171-196). Cambridge: MIT Press.

Futo, J., Teglas, E., Csibra, G., \& Gergely, G. (2010). Communicative function demonstration induces kind-based artifact representation in preverbal infants. Cognition, 117, 1-8.

Gelman, R. (1990). First principles organize attention to relevant data: Number and the animate-inanimate distinction as examples. Cognitive Science, 14, 79-106.

Gelman, S. A. (2003). The essential child: Origins of essentialism in everyday thought. New York: Oxford University Press.

Gelman, R. (2009). Learning in core and noncore domains. In L. Tommasi, M. A. Peterson, \& L. Nadel (Eds.), Cognitive Biology (pp. 247-260). Cambridge: The MIT Press.

Gelman, S. A., \& Gottfried, G. D. (1996). Causal explanations of animate and inanimate motion. Child Development, 67, 1970-1987.

Gelman, S. A., \& Hirschfeld, L. A. (1999). How biological is essentialism? In D. L. Medin \& S. Atran (Eds.), Folkbiology (pp. 403-446). Cambridge: The MIT Press.

Gelman, S. A., \& Markman, E. M. (1986). Categories and induction in young children. Cognition, 23, 183-209.

Gelman, S. A., \& Wellman, H. M. (1991). Insides and essences: Early understanding of the non-obvious. Cognition, 38, 213-244.

Gergely, G., \& Csibra, G. (2003). Teleological reasoning in infancy: The naive theory of rational action. Trends in Cognitive Sciences, 7, 287 292.

Gergely, G., \& Jacob, P. (2012). Reasoning about Instrumental and Communicative Agency in Human Infancy. In J. B. Benson, F. Xu, T. Kushnir, \& J. B. Benson (Eds.), Rational Constructivism in Cognitive Development (pp. 59-94). Academic Press: Elsevier Inc.

Gopnik, A., \& Nazzi, T. (2003). Words, kinds, and causal powers: a theory theory perspective on early naming and categorization. In 
D. H. Rakison \& L. M. Oakes (Eds.), Early Category and Concept Development. Making sense of the blooming buzzing confusion. USA: Oxford University Press.

Gopnik, A., \& Sobel, D. M. (2000). Detecting blickets: How young children use information about novel causal powers in categorization and induction. Child Development, 71, 1205-1222.

Gottfried, G. M., \& Gelman, S. A. (2005). Developing domain-specific causal-explanatory frameworks: The role of insides and immanence. Cognitive Development, 20, 137-158.

Gottfried, G., Gelman, S., \& Schultz, J. (1999). Children's understanding of the brain: From early essentialism to biological theory. Cognitive Development, 14(1), 147-174.

Graham, S. A., \& Diesendruck, G. (2010). Fifteen-month-old infants attend to shape over other perceptual properties in an induction task. Cognitive Development, 25, 111-123.

Hamlin, J. K., Wynn, K., \& Bloom, P. (2007). Social evaluation by preverbal infants. Nature, 450, 557-560.

Hatano, G., \& Inagaki, K. (1999). A developmental perspective on informal biology. In D. L. Medin \& S. Atran (Eds.), Folkbiology (pp. 321-354). Cambridge: The MIT Press.

Hernik, M., \& Csibra, G. (2009). Functional understanding facilitates learning about tools in human children. Current Opinion in Neurobiology, 19, 34-38.

Heyman, G. D., \& Gelman, S. A. (2000). Beliefs about the origins of human psychological traits. Developmental Psychology, 36(5), 663.

Hirsch, E. (1982). The concept of identity. New York: Oxford University Press.

Inagaki, K., \& Hatano, G. (1993). Young children's understanding of the mind-body distinction. Child Development, 64, 1534-1549.

Inagaki, K., \& Hatano, G. (2002). Young children's naive thinking about the biological world. New York: Psychology Press.

Inagaki, K., \& Hatano, G. (2004). Vitalistic causality in young children's naïve biology. Trends in Cognitive Sciences, 8(8), 356-362.

Johnson, S., \& Carey, S. (1998). Knowledge enrichment and conceptual change in folkbiology: Evidence from Williams Syndrome. Cognitive Psychology, 37, 156-200.

Keil, F. (1989). Concepts, Kinds, and Cognitive Development. Cambridge: MIT Press.

Keil, F.C. (1991). The Emergence of Theoretical Beliefs as Constraints on Concepts. In S. Carey and R. Gelman (Eds.), The Epigenesis of Mind: Essays on Biology and Cognition (pp. 237-256). Earlbaum

Keil, F. C. (1995). The Growth of Causal Understandings of Natural Kinds: Modes of Construal and the Emergence of Biological Thought. In A. Premack \& D. Sperber (Eds.), Causal Cognition. Oxford: Oxford University Press.

Kelemen, D., \& Carey, S. (2007). The essence of artifacts: Developing the design stance. In E. Margolis \& S. Laurence (Eds.), Creations of the Mind: Theories of artifacts and their representation (pp. 212-230). USA: Oxford University Press.

Kingo, O. S., \& Krojgaard, P. (2011). Object manipulation facilitates kind-based object individuation of shape-similar objects. Cognitive Development, 26(2), 103-111.

Kripke, S. (1980). Naming and necessity. Cambridge: Harvard University Press.

Kuhlmeier, V., Wynn, K., \& Bloom, P. (2003). Attribution of dispositional states by 12 -month-olds. Journal of Cognitive Neuroscience, 14(5), 402-408.

Leslie, A. M. (1984). Spatiotemporal continuity and the perception of causality in infants. Perception, 13, 287-305.

Leslie, A. M. (1994). ToMM, ToBy, and agency: Core architecture and domain specificity. In L. Hirschfeld \& S. Gelman (Eds.), Mapping the mind: Domain specificity in cognition and culture (pp. 119148). New York: Cambridge University Press.

Mandler, J. M. (2004). The foundations of Mind: Origins of conceptual thought. USA: Oxford University Press.
Mandler, J. M., \& McDonough, L. (1993). Concept formation in infancy. Cognitive Development, 8, 291-318.

Mandler, J. M., \& McDonough, L. (1998). Studies in inductive inference in infancy. Cognitive Psychology, 37, 60-96.

Medin, D. L., \& Atran, S. (1999). Folkbiology. Cambridge: Bradford.

Medin, D. L., \& Ortony, A. (1989). Psychological essentialism. In S. Vosniadou and A. Ortony (Eds.), Similarity and analogical reasoning. New York: Cambridge University Press.

Meunier, B., \& Cordier, F. (2009). The role of feature type and causal status in 4-5-years-old children's biological categorizations. Cognitive Development, 24, 34-48.

Morris, S. C., Taplin, J. E., \& Gelman, S. (2000). Vitalism in naïve biological thinking. Developmental Psychology, 36(5), 582-595.

Newman, G. E., Herrmann, P., Wynn, K., \& Keil, F. C. (2008). Biases towards internal features infants' reasoning about objects. Cognition, 107, 420-432.

Newman, G., \& Keil, F. (2008). Where is the essence? Development shifts in children's beliefs about internal features. Child Development, 79(5), 1344-1356.

Opfer, J. E. (2002). Identifying living and sentient kinds from dynamic information: The case of goal-directed versus aimless autonomous movement in conceptual change. Cognition, 86, 97-122.

Opfer, J. E., \& Siegler, R. (2004). Revisiting preschoolers' living things concept: A microgenetic analysis of conceptual change in basic biology. Cognitive Psychology, 49, 301-332.

Pauen, S. (2002). Evidence for knowledge-based category discrimination in infancy. Child Development, 73(4), 1016-1033.

Prasada, S., Hennefield, L., \& Otap, D. (2012). Conceptual and linguistic representations of kinds and classes. Cognitive Science, 36, 1224 1250

Putnam, H. (1975). Mind, Language, and Reality. New York: Cambridge University Press.

Quine, W. V. (1969). Natural kinds. In W. V. Quine (Ed.), Ontological relativity and other essays (pp. 114-138). New York: Columbia University Press.

Rehder, B. (2003). Categorization as causal reasoning. Cognitive Science, 27, 709-748.

Rehder, B. (2007). Essentialism as a generative theory of classification. In A. Gopnik \& L. Schultz (Eds.), Causal learning: Psychology, philosophy, and computation (pp. 190-207). Oxford: Oxford University Press.

Rehder, B., \& Kim, S. (2006). How causal knowledge affects classification: A generative theory of categorization. Journal of Experimental Psychology: Learning, Memory, and Cognition, 32, 59-683.

Rehder, B., \& Kim, S. W. (2009). Classification as diagnostic reasoning. Memory \& Cognition, 37, 715-729.

Rhodes, M., \& Gelman, S. A. (2009). A developmental examination of the conceptual structure of animal, artifact, and human social categories across two cultural contexts. Cognitive Psychology, 59, 244-274.

Rips, L. J. (2011). Lines of Thought: Central concepts in cognitive psychology. USA: Oxford University Press.

Setoh, P., Wu, D., Baillargeon, R., \& Gelman, R. (2013). Young infants have biological expectations about animals. Proceedings of the National Academy of Sciences, 110(40), 15937-15942.

Shipley, E. F. (1989). Two kinds of hierarchies: Class inclusion hierarchies and kind hierarchies. The Genetic Epistemologist, 17, 31-39.

Simons, D., \& Keil, F. C. (1995). An abstract to concrete shift in the development of biological thought: The insides story. Cognition, 56, 129-163.

Sobel, D. M., \& Buchanan, D. W. (2009). Bridging the gap: Causality-ata-distance in children's categorization and inferences about internal properties. Cognitive Development, 24, 274-283.

Sobel, D. M., Yoachim, C. M., Gopnick, A., Meltzoff, A. N., \& Blumenthal, E. J. (2007). The Blicket Within: Preschoolers' inferences about insides and causes. Journal of Cognition and Development, 8(2), 159-182. 
Springer, K., \& Keil, F. C. (1991). Early differentiation of causal mechanisms appropriate to biological and nonbiological kinds. Child Development, 62, 767-781.

Surian, L., \& Caldi, S. (2010). Infant's individuation of agents and inert objects. Developmental Science, 13(1), 143-150.

Taborda-Osorio, H. \& Cheries, W. E. (2015). Infants' agent individuation: It's what's on the insides that counts. Manuscript submitted for publication.

Taborda-Osorio, H. \& Cheries, W. E. (2016). Individuation of agents by moral dispositions. Manuscript in preparation.

Taborda-Osorio, H., Lyons, A., \& Cheries, W. E. (2016). Insides and moral dispositions. Manuscript in preparation.

Tenenbaum, J. B., Griffiths, T. L., \& Kemp, C. (2006). Theory-based Bayesian models of inductive learning and reasoning. Trends in Cognitive Sciences, 10(7), 309-318.

Trauble, B., \& Pauen, S. (2007). The role of functional information for infant categorization. Cognition, 105, 362-379.

Van de Walle, G. A., Carey, S., \& Prevor, M. (2000). Bases of object individuation in infancy: Evidence from manual search. Journal of Cognition and Development, 1(3), 249-280.
Ware, E. A., \& Booth, A. E. (2010). Form follows function: The role of artifact function in the development of the shape bias. Cognitive Development, 25, 124-137.

Waxman, S., Medin, D., \& Ross, N. (2007). Folkbiological reasoning from a cross-cultural developmental perspective: Early essentialist notions are shaped by cultural beliefs. Developmental Psychology, 43(2), 294-308.

Welder, A. N., \& Graham, S. A. (2006). Infants' categorization of novel objects with more or less obvious features. Cognitive Psychology, $52,57-91$.

$\mathrm{Xu}, \mathrm{F}$. (2005). Categories, kinds, and object individuation in infancy. In L. Gershkoff-Stowe \& D. Rakison (Eds.), Building object categories in developmental time (pp. 63-89). Papers from the 32nd Carnegie Symposium on Cognition. New Jersey: Lawrence Erlbaum.

Xu, F., \& Carey, S. (1996). Infant's metaphysics: The case of numerical identity. Cognitive Psychology, 30, 111-153.

Xu, F., Carey, S., \& Quint, N. (2004). The emergence of kind-based object individuation in infancy. Cognitive Psychology, 49, 155-190. 